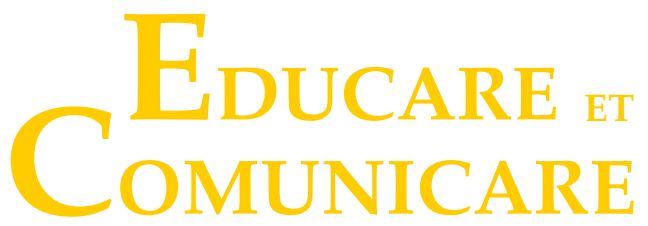

REVISTA CIENTÍFICA DE LA FACULTAD DE HUMANIDADES

https://DOI 10.35383/educare.v9i2.583

e-ISSN: 2312-9018

Universidad Católica Santo Toribio de Mogrovejo

\title{
Pedagogía de la ternura para disminuir conductas agresivas en niños
}

\section{Pedagogy of tenderness to reduce aggressive behavior in children}

\author{
Carmen Cajusol Santisteban ${ }^{* 1 a}$ \\ ccajusols@ucvvirtual.edu.pe \\ https://orcid.org/0000-0002-0535-3011
}

Fiorela Anaí Fernández-Otoya ${ }^{2 a}$

ffernandez@usat.edu.pe

https://orcid.org/0000-0003-0971-335X

\author{
*Autor corresponsal \\ 1 Universidad César Vallejo, Chiclayo, Perú \\ 2 Universidad Católica Santo Toribio de Mogrovejo, \\ Chiclayo, Perú \\ a Doctor de Educación
}

\section{Fechas importantes}

Recibido: $17 / 05 / 2021$

Aceptado:31/07/2021

Publicado online: 31/12/2021

\section{Resumen}

El presente artículo nos describe los resultados de la puesta en práctica de un programa de pedagogía que se basa en ternura, la cual se aplicó en dos grupos de niños del nivel inicial con el fin de descubrir el impacto positivo en la disminución de manifestaciones agresivas que los niños presentaban. Por consiguiente, el objetivo puso concentración en determinar el impacto del programa de pegagogía en base a ternura para constrarrestrar las conductas agresivas en niños y niñas de las I.E.I N ${ }^{\circ} 313$ Héctor Aurich del distrito de Ferreñafe y I.E.I San Gabriel N ${ }^{\circ}$ 051 del distrito de Pomalca. Según la metologogía de la investigación, fue aplicativa con diseño cuasi experimental, donde se utilizó como herramientas de recolección de información un pre y post test en la muestra de estudio evaluando la variable dependiente de agresividad. No obstante, se tomó como muestra a toda la población por ser reducida y tener acceso a ellos siendo 180 niñas y niños de las dos instituciones antes mencionadas. El programa se fundamenta principalmente en talleres donde los principles involucrados para erradicar el problema son los padres de los niños logrando un resultados exitosos disminuyendo la cifra de agresividad física encontrada de un $57 \%$ a un $23 \%$. Asimismo, las conductas agresivas verbales redujeron de un $76 \%$ a un $30 \%$ y por último, de un $66 \%$ a $26 \%$ el comportamiento psicológico agresivo. Finalmente, los programas de pedagogía son importantes herramientas que generan resultados con éxito, si los padres y docentes lo aplican continuamente mostrando sensibilidad, interés y responsabilidad por guiar con amor y en valores, permitiéndoles crecer sanos y felices.

Palabras clave: pedagogía de la ternura, conductas agresivas, programa de pedagogía 


\begin{abstract}
This article describes the results of the implementation of a pedagogy program that is based on tenderness, which was applied in two groups of children of the initial level in order to discover the positive impact on the reduction of aggressive manifestations that children presented. Therefore, the objective focused on determining the impact of the pedagogy program based on tenderness to counteract aggressive behaviors in boys and girls from IEI N ${ }^{\circ} 313$ Héctor Aurich from the district of Ferreñafe and IEI San Gabriel ${ }^{\circ} 051$ from the district of Pomalca. According to the research methodology, it was an application with a quasi-experimental design, where a pre and post test was used as information collection tools in the study sample, evaluating the dependent variable of aggressiveness. However, the entire population was taken as a sample because it was small and had access to them, being 180 girls and boys from the two aforementioned institutions. The program is based mainly on workshops where the principles involved to eradicate the problem are the parents of the children, achieving successful results, reducing the number of physical aggression found from $57 \%$ to $23 \%$. Likewise, verbal aggressive behaviors reduced from $76 \%$ to $30 \%$ and finally, from $66 \%$ to $26 \%$, aggressive psychological behavior. Finally, pedagogy programs are important tools that generate successful results, if parents and teachers apply them continuously, showing sensitivity, interest and responsibility to guide with love and values, allowing them to grow up healthy and happy.
\end{abstract}

Keywords: pedagogy of tenderness, aggressive behaviors, pedagogy program

\title{
Introducción
}

La ONU (2017) dio detalles que un promedio de 32\% de alumos son intimidados por sus demás compañeros o violentados físicamente. Por otra parte, el MINEDU (2018), dio a conocer que mayormente las agresiones físicas son más comunes en los colegios y un $8 \%$ de la población estudiantil agresiva son niños del nivel inicial lo que conlleva a generar preocupación que los padres no están pestando la debida y adecuada atención a sus niños por lo que ellos, muestran en las escuelas, hay un promedio de $32 \%$ de alumos son intimidados por sus demás compañeros o violentados físicamente deficiencias existentes en sus hogares. También, el Diario Perú 21 (2017) menciona distintas razones por la que los niños muestran conductas agresivas en las escuelas y una de ellas es la ruptura de matrimonio de los padres puesto que los padres muchas veces, mezclan la relación de pareja con la de padres; sin embargo, los quehaceres son diferentes. Ambos deben seguir cumpliendo con su rol lleno de amor poque ellos no tienen por qué presenciar el distanciamiento hacia el niño por parte de sus padres y aún mucho más, si el niño evidencia discusiones entre ellos por gozar por separado a sus hijos.

Por otra parte, la Organización Mundial de la Salud (2019) ofrece consejos para prevenir la agresividad en niños de las edades de 3 a 5 , cuyos consejos no solo se direccionan a los padres sino también a los maestros del nivel inicial. Y las recomendaciones abordan que lo primero que se debe hacer en la escuela es prestar la debida atención en el compartamiento que traen los niños desde casa e ir plasmándolos en una hoja de ruta para darles seguimiento a cada uno de ellos. Ante ello, cabe describir la problemática evidenciada en los niños y niñas de las I.E I N 313 localizada en la ciudad de Ferreñafe sector Hector Aurich donde los niños reflejan claras actitudes agresivas de niños hacia sus compañeros. Por otra parte, este problema también es claro en la I.E.I de Pomalca donde los niños usan mucho las rabietas y expresan rabia empujando a los demás, mordiendolos, insultándolos e intimidándolos. Claros comportamientos agresivos se deben enlazar con el escaso interés que los padres tienen hacia sus hijos, puesto que más importancia tiene para ellos el tener en sus boletas de notas buenas calificaciones que el buen desarrollo socioemocional de sus niños.

Los niños agresivos tienen más probabilidades de experimentar enfermedades físicas y mentales, desempleo, pobreza y problemas forenses cuando sean adultos. Las estrategias para prevenir la agresión deben abordar los factores de riesgo: baja educación materna, embarazo adolescente, exposición intrauterina al tabaco y al alcohol, pobreza, crianza coercitiva, maltrato infantil y victimización por bullying. 
El niño agresivo requiere una evaluación exhaustiva para identificar y manejar problemas subyacentes o comórbidos, para comprender las razones del comportamiento agresivo y para detectar factores modificables que exacerban o perpetúan la agresión. (Cavell et al., 2020, p.5). La presente investigación parte por considerar talleres donde se solicitó la participación de los padres porque es en su hogar donde se forman o se moldean ciertos tipos de conductas que el niño reflejo y por lo tanto, ellos son quienes deben ayudar a sus niños a erradicar esas conductas con amor.

En primer lugar, Repo y Sajaniemi (2015) en su investigación abordó que la intimidación empieza generalmente a temprana edad de los 3 a 6 años. Además, señala que la intimidación afecta directamente la autoestima del niño. Sin embargo, la prevalencia en la intimidación se logra disminuir abordando estrategias educativas especiales en sus relaciones con los compañeros (p.72). Asimismo, Quiñones et al. (2018) abordan que en los entornos virtuales también puede demostrar afecto a los niños, decirles palabras dulces y sinceras permitirá que el niño se sienta motivado y por tanto, disminuya la angustia y presión del entorno. Cuando los docentes, padres o tutores le brindan seguimiento al niño y se interesan por ellos su autoestima se eleva. Del mismo modo, es importante concientizar a todas las escuelas e instituciones para dar a conocer que las demostraciones de amor a los niños no solo se pueden realizar físicamente sino aprovechando la tecnología para aprender y nutrir el aspecto psicológico de niños y niñas. Por ende, hay que fomentar el compromiso de incorporar las TIC junto con una capacitación para desarrollar competencias afectivas en los profesores (p. 25). Mientras se aproveche en alimentar y nutrir a los niños de afecto sincero y amor, se afianzará en ellos la seguridad y confianza donde ellos tomarán la iniciativa de aprender de forma presencial y virtual. Esta labor deben iniciarla los docentes que son conocedores de estrategias para guiar a los padres y niños a interactuar siempre.

Por último, Sánchez (2018) indican que las razones por la que los niños manifiestan conductas agresivas es porque los padres son duros con ellos en la forma de educarlos, observan conflictos familiares agresivos dentro de la familia o generalmente los familiares no le prestan la debida atención. Asimismo, Ferreyra (2016) destaca que los padres por las ansias que los niños cumplan con sus tareas escolares reducen los tiempos de juego y presionan par cumplir con el contenido pedagógico, estas acciones conllevan a aumentar las conductas agresivas de los niños y las acciones claras para enfrentar estos problemas es dedicar tiempo a los niños, jugar con ellos, usar técnicas de relajación y un auxilar del maestro en la escuela ayuda a poder atender mas a precisión las necesidades de los niños.

\section{Pedagogía de la ternura}

Cussiánovich la define como la capacidad que tienen las personas para expresar amor, empleado distintos tipos de estrategias para impartirla y crear claros ambientes de amornía usando metódos lúdicos. Expresa que esta capacidad permite quererse y amarse a uno mismo construyendo una mejor autoestima, una identidad, mayor capacidad para relacionarse, y podrá encontrarle sentido a la propia vida. En el ámbito educativo y pedagógico, si no se establece un vínculo constructivo y positivo, no hay proceso educativo. (Cussiánovich, 2015, p.63). La ternura atribuye cualidades amorosas que impulsan a querer, a cultivar la sensibilidad, que necesita de responsabilidad, de afectos amorosos, desde la niñez. Sin embargo, en la infancia tienden a pelear por las posesiones; Aprender a controlar sus emocioneses un desafío en esta etapa. Los berrinches son muy frecuentes y a veces van con agresión. En la niñez usan la agresión para llamar la atención. Los infantes imitan a otros. "iNo!" Es una palabra que los pequeños utilizan para afirmar su creciente independencia. Mientras que la agresión física aumenta con la edad, lo importantees que apartir de los 3 años laagresión física debería de disminuir. (Maya, 2015, p.81).

Según Cruz (2017) la pedagogía tiene como dimensiones a: La afectividad que la define como la necesidad que tenemos los seres humanos de establecer lazos con otras personas. El amor también es una dimensión que al expresarlo hacia los niños, su cerebro se activa a producir hormonas de felicidad por lo que es importante para cultivar seguridad en sí mismos y permite que cada etapa de su vida se desarrolle adecuadamente. Asimismo, otra dimesión a la que se sujeta nuestra investigación es la sensibilidad la cual es 
la forma de sentir dulce para responder adecuadamente a las emociones, cabe señalar que para desarrollarla es importante atender el sentir que tiene cada niño lo que nos permitirá encontrar y desarrollar sus talentos, vocación y sus pasiones por algunas cosas. Por último, la amabilidad es un valor social que se funda en el respeto, el afecto y benevolencia en nuestra forma de relacionarnos con el otro. La amabilidad es esencial para la convivencia en sociedad. No es innata del niño, pues éste es impulsivo por naturaleza y el ser amable y cortes se aprende en la realización de actividades de la vida cotidiana. (p. 103).

\section{Agresividad}

En cuanto a las conductas de agresividad que los niños muestren hacia sus compañeros, Juul (2015) revela que las conductas agresivas mostradas por los infantes son muestra de la falta de cariño de sus padres o su entorno. Por esta razón, los pequeños parecen ser propensos a sentirse cada vez más frustrados por la falta de habilidades de afrontamiento o de vocabulario para expresarse adecuadamente. Por ello, los niños tienden a generar sentimientos de ira o alguna conducta inadecuada y los padres o apoderados en lugar de ayudar a identificar el por qué de sus sentimientos hacen caso omiso a estas actitudes. Sin duda, que la mejor terapia para erradicar las conductas agresivas son la afectividad, el amor, la ternura y amabilidad. Por lo tanto, brindar amor a los niños involucra mejoras en el aprendizaje, buena memoria, buen control de las emociones y eliminar el estrés. Cabe mencionar, que los niños cargados de estrés están cargados de emociones tóxicas tanto que afectan su salud y las de su entorno. El buen ejemplo y el entrenamiento adecuado moldeará de la mejor forma al niño y cuya formación se evidenciará en su conducta.

Existen formas de adquirir la agresividad, desde la perspectiva de Bandura (1975) analizó la adquisición de agresividad mediante el aprendizaje, el cual abordó que los niños aprenden mediante la observación y experiencia. Por ejemplo, si el niño evidencia constantes escenas de agresión en su familia o su entorno es acertado que éste adquiera tales conductas. Por otro lado, el estrés y la frustración instigan a los niños a ser agresivos. Por ello, ni bien se detecte este tipo de manifestaciones se debe abordar acciones de mejora.

\section{Metodología}

El diseño de la presente investigación fue cuasi experimental aplicando las herramientas del pretest y postest donde el programa de pedagogía gira entorno al grupo experimental. El enfoque de la investigación es cuantitativo porque mide los datos recolectados y por ende, han sido validados respectivamente por doctores en Educación.

Debido al acceso de la información, el número de individuos aplicada en la población fue la misma tomada en la muestra. Del total de la muestra, 90 niños conforman el grupo experimental direccionada a la I.E.I N 313- Héctor Aurich y por otro lado, el grupo de control lo conformaron 90 niños de la I. E.I San Gabriel N 051 de Pomalca. La población tomada estuvo matriculada y asistieron con regularidad. La lista de cotejo se realizó como recolección de información sobre la variable dependiente sobre agresividad en los niños y proponer la metodología de la investigación y realizar las actividades diagnósticas para evaluar las áreas deficientes de estudio. Además se hizo uso de la técnica documental para recolectar la información recabada de diferentes libros y folletos proporcionados en clases y páginas Web. También, se utilizó la técnica de campo que fue usada para confrontar la teoría y la realidad. Las herramientas daves en esta investigación han sido el cuestionario. Se trabajó con los padres de familia y niños. Los talleres basados en amor también enriquecieron este análisis. Este instrumento principal de recolección de información fue estructurado dentro del programa pedagógico en base a ternura con 12 ítems para explorar el efecto que tieneaplicarlo para disminuir las conductas agresivas en niños y padres; losítems fueron de carácter cerrado.

El instrumento evaluó la variable de agresividad en sus tres dimensiones: física, verbal y psicológica. Es importante señalar que en agresividad física se manifiesta con conductas violentas donde se aplica las interrogantes ¿EI niño pelea con sus compañero? También, ¿El niño le golpea o muerde?, cuando el agresor emplea la violencia directa, éste ataca a su víctima en confrontación abierta, para ello utiliza los 
ataques físicos, amenaza, rechazo y destrucción de la propiedad del otro. En este indicador interrogó si el niño golpea a su compañero cuando este no le da lo que le pide donde se recogerá información para analizarla y mediante estrategias abordar este problema. Por otro lado, se desea saber si los estudiantes tiran objetos a la maestra o a sus compañeros. Por otro lado, la disrupción se manifiestan en rabietas para llamar la atención a los padres o maestros donde estas conductas son inadecuadas más si se presentan en las aulas donde los demás niños evidencias las conductas de sus demás compañeros e inician a copiarlas. Un indicador como este permite evaluar si cuando un niño golpea a otro, él hace lo mismo o si cuando el docente le quita la vista por unos momentos, éste aprovecha a golpear a sus compañeros o si cuando el niño esta enojado se desquita rompiendo objetos.

En la dimensión verbal examina ítems sobre las manifestaciones de descargas emocionales usando palabras con respuestas habladas ya sea emitiendo insultos, amenazas a sus compañeros o rechazos hacia ellos. Los insultos son un tipo de intimidación que causan mucho daño porque las víctimas insertan en su ser esas palabras de dolor afectando su autoestima. Por ello, es importante considerar los ítems que evalúan si existe violencia directa en el entorno preguntando si el niño genera burlas a sus compañeros, si le coloca apodos o si se fija en los defectos de ellos. En el indicador de ansiedad se debe analizar si el niño o niña emite palabras soeces en el aula o si cuando otro compañero lo insulta él también hace lo mismo.

Cabe iniciar que en la dimensión psicológica, se toma en cuenta que las conductas agresivas presentadas pretenden ir contra el autoestima de los demás empleando acciones como los murmullos, lanzar adjetivos, mala información o calumnias que van a afectar directamente al niño. En esta dimensión se evalúa si el niño genera desprecio contra sus otros compañeros que son de menor tamaño o menor fuerza. Además, si disfruta haciendo llorar o intimidando a sus compañeros, o si éste le gusta amenazar a otros. Por otra parte, se toma el indicador de seguridad si el niño lanza mentiras sobre sus demás compañeros y mediante el indicador de inadaptación se consulta si el niño agresivo tiene problemas con otros de sus compañeros en el aula o si el niño es golpeado también por los demás. Por último, se necesita saber si el niño le arrebata las cosas a otros compañeros por generar molestas en ellos.

\section{Resultados}

\section{Nivel de agresividad inicial}

A continuación se presentan los resultados obtenidos a través del pretest realizado a través de la observación directa a los dos grupos en evaluación.

\section{Tabla 1}

El niño muerde o golpea a sus compañeros

\section{GRUPOS PRETEST}

\begin{tabular}{ccccc} 
& \multicolumn{4}{c}{ GRUPOS PRETEST } \\
\cline { 2 - 5 } Ítem & \multicolumn{2}{c}{ EXPERIMENTAL-FERREÑAFE } & \multicolumn{2}{c}{ CONTROL-POMALCA } \\
\cline { 2 - 5 } & $\mathbf{N}^{\circ}$ & $43 \%$ & 40 & $\mathbf{\%}$ \\
\hline No & 39 & $57 \%$ & 50 & $56 \%$ \\
Si & 51 & $\mathbf{1 0 0 \%}$ & $\mathbf{9 0}$ & $\mathbf{1 0 0 \%}$ \\
Total & $\mathbf{9 0}$ & &
\end{tabular}

En la Tabla 1, se halló que en ambos grupos la agresividad física en los niños la manifiesta nn mediante golpes o mordidas a otros compañeros resultando un índice del $57 \%$ y $56 \%$ en el grupo experimental Ferreñafe y en control Pomalca. Para intervenir estos problemas, el docente y los padres de familia deben observar el por qué sus hijos realizan estas acciones que hacen daño a los demás, para ellos se necesita mucha paciencia de los padres para tratar con amor y erradicar estas acciones fomentando las conductas positivas que tienen sus hijos. Por otra parte, un $43 \%$ y $44 \%$ de los niños en evaluación no presentan este tipo de conductas agresivas físicas. 
Tabla 2

Retribuición de insultos al compañero que los insulta

\begin{tabular}{ccccc}
\hline & \multicolumn{4}{c}{ GRUPOS PRETEST } \\
\cline { 2 - 5 } Ítem & $\mathbf{N}^{\circ}$ & $\mathbf{\%}$ & $\mathbf{N}^{\circ}$ & $\mathbf{\%}$ \\
\cline { 2 - 5 } & 22 & $24 \%$ & 25 & $28 \%$ \\
\cline { 2 - 4 } No & 68 & $76 \%$ & 65 & $72 \%$ \\
Si & $\mathbf{9 0}$ & $\mathbf{1 0 0 \%}$ & $\mathbf{9 0}$ & $\mathbf{1 0 0 \%}$ \\
\hline
\end{tabular}

Consecutivamente, para la evaluación de la dimensión de agresión verbal, tomamos como referencia el ítem que indica si el niño insulta a sus compañeros, cuyos datos nos arrojan que el $76 \%$ y $72 \%$ realizan estas conductas agresivas verbales siendo un índice elevado en un aula de niños del nivel inicial. Debemos recordar que los insultos que ellos emiten mayormente se generan porque escuchan a los adultos de casa o a las personas cercanas. Ellos no entienden el significado de los insultos pero si se graban en sus mentes el contexto y la forma que lo expresan las personas de quienes han escuchado estas acciones. Cuando abordamos estas situaciones debemos empezar por mostrarles como se sienten los demás cuando los insultan porque ellos mismos deben darse cuenta y aprender que estas acciones lastiman a los demás.

\section{Tabla 3}

Amenazas hacia los compañeros

\section{GRUPOS PRETEST}

\begin{tabular}{ccccc}
\multirow{2}{*}{ Ítem } & \multicolumn{2}{c}{ EXPERIMENTAL-FERRENAFE } & \multicolumn{2}{c}{ CONTROL-POMALCA } \\
\cline { 2 - 5 } & $\mathbf{N}^{\circ}$ & $\mathbf{\%}$ & $\mathbf{N}^{\circ}$ & $\mathbf{\%}$ \\
\hline No & 26 & $29 \%$ & 29 & $32 \%$ \\
Si & 64 & $71 \%$ & 61 & $68 \%$ \\
Total & $\mathbf{9 0}$ & $\mathbf{1 0 0 \%}$ & $\mathbf{9 0}$ & $\mathbf{1 0 0 \%}$ \\
\hline
\end{tabular}

Por último, en la Tabla 3 se puede analizar que hay indicios elevados de violencia psicológica de un $71 \%$ y $68 \%$ en el grupo de Ferreñafe y Pomalca respectivamente. Estas cifras son alarma que están creciendo en un lugar con escaso de amor, donde indicios de soledad los conllevan a cargarse de emociones negativas que so descargadas generalmente en niños con emociones de alegría y felicidad generando un cierto miedo en ellos puesto que no se han rodeado de ambientes tóxicos antes. En el grupo de control existe una diferencia del 3\% respecto al grupo experimental. En la aplicación del programa se tuvo en consideración las dimensiones que abordan la pedagogía de la ternura.

\section{Aplicación del programa Pedagogía de la ternura}

Las 12 sesiones de aprendizaje aplicadas se basaron a la pedagogía de la ternura. Se diseñó el programa y se consideraron los datos arrojados en el pretest del diagnóstico de agresividad para abordar sesiones referentes a disminuir a la agresividad. Por ello, se trabajó la dimensión del afecto, constituida por 3 sesiones: la primera titulada Valora la amistad, utilizando 30 minutos para abordar dicha sesión. Se enriqueció con dinámica y canciones con el fin de familiarizar a todos los niños. Seguidamente, se consideró la sesión: Actúa en base a valores. Se abordó el tema de valores porque al enseñarles y practicarlos, los niños mediante la experiencia y la visión adoptarán mejores comportamientos. Por último, 
habla con dulces palabras. Esta sesión permitió a los niños expresar mediante la dinámica del espejo, lo que sienten e reflejarse con cada sentimiento de sus compañeros.

Después de haber aplicado nuestra lista de cotejo, se diseñó talleres aplicadas en 12 sesiones. Los talleres consistieron en buscar la participación de los padres inculcando en sus hijos el amor, la ternura, la paciencia y comprensión. La participación de ellos es tan primordial pues se busca activamente socializarnos sin miedo, promoviendo no sólo el enriquecimiento de conocimiento sino la formación de niños saludables llenos de amor en compañía de sus padres. El tema principal de todos los talleres fue enseñar en base a ternura y amor. Se buscó intensamente disminuir la agresividad en ellos.

Para el desarrollo del taller, el rol de la maestra produce familiaridad y seguridad en la convivencia en el aula, además; valorar y escuchar a sus niños y niñas permite que se sientan que tienen una maestra que les ayude en cada instante, apreciando lo que valen como seres humanos. Hacer que se sientan aceptados y aceptarse entre ellos por ser únicos, iguales ante la ley, pero diferentes en cualidades. Crear respeto les enseñara a tratarnos bien como elementos de un solo ambiente estudiantil. Seguidamente, generar motivación los tendrán activos y aptos para aprender todos juntos y por último; generar relaciones cordiales y afectuosas, brindándonos ternura entre todos. Por ello, abrazar a sus padres y dibujarlos hará crecer en ellos el amor fraterno exponiendo lo que le gusta de su padre y que le gusta hacer con ellos o ellas.

Realizada la aplicación del programa de pedagogía en base a ternura, ejecutado en 12 talleres o sesiones de aprendizaje, se procedió a evaluar nuevos diagnósticos mediante un postest, con los mismos ítems del pretest para verificar y analizar los efectos que se han logrado; por lo tanto, la información recopilada indica que los niveles de agresividad en la Institución Educativa $N^{\circ} 313$ Héctor Aurich perteneciente al grupo experimental, de los cuales se resume en lo siguiente:

\section{Nivel de agresividad final}

En las Tabla 4, 5 y 6 se muestran el nivel de agresividad en niños de las Instituciones Educativas de Inicial de los distritos de Ferreñafe y Pomalca, adquirido en el postest, según sus dimensiones física, verbal y psicológica.

\section{Tabla 4}

El niño le gusta morder o golpear a sus compañeros

\section{GRUPOS POSTEST}

\begin{tabular}{ccccc} 
Ítem & \multicolumn{2}{c}{ EXPERIMENTAL-FERRENAFE } & \multicolumn{2}{c}{ CONTROL-POMALCA } \\
\cline { 2 - 4 } & $\mathbf{N}^{\circ}$ & $\mathbf{\%}$ & $\mathbf{N}^{\circ}$ & \multicolumn{1}{c}{ \% } \\
\hline No & 69 & $77 \%$ & 40 & $56 \%$ \\
Si & 21 & $23 \%$ & 50 & $\mathbf{1 0 0 \%}$ \\
\hline
\end{tabular}

Mediante esta tabla se analiza si el niño después de la aplicación de talleres aún persiste en ellos las conductas agresivas físicas, por lo que se obtiene una disminución del $33 \%$ respecto al grupo de control. Por tanto, el índice se encuentra en un $23 \%$. Según las teorías anteriormente analizadas, hacen referencia que un niño agresivo es un ser que está lleno de fustración y libera el desiquilibrio de sus emociones en mordidas o en otras conductas agresivas. Las herramientas aplicadas han sido eficientes para eliminar las conductas agresivas de los niños. Ahora es tarea de los padres que sigan moldeando con amor a sus pequeños para que a donde vayan demuestren su felicidad transformada en energía. Los docentes deben continuar esta ardua labor de educar con amor, mucha mas paciencia y dedicación a niños que solo expresan los que están viviendo en su alredor de distintas formas positivas o negativas. 
Tabla 5

Retribución de insultos a sus compañeros

\begin{tabular}{ccccc}
\hline & \multicolumn{4}{c}{ GRUPOS POSTEST } \\
\cline { 2 - 5 } Ítem & \multicolumn{2}{c}{$\begin{array}{c}\text { EXPERIMENTAL- } \\
\text { FERRENAFE }\end{array}$} & CONTROL-POMALCA \\
\cline { 2 - 5 } & $\mathbf{N}^{\circ}$ & $\mathbf{\%}$ & $\mathbf{N}^{\circ}$ & $\%$ \\
\cline { 2 - 5 } No & 58 & $64 \%$ & 25 & $28 \%$ \\
Si & 32 & $36 \%$ & 65 & $72 \%$ \\
Total & $\mathbf{9 0}$ & $\mathbf{1 0 0 \%}$ & $\mathbf{9 0}$ & $\mathbf{1 0 0 \%}$ \\
\hline
\end{tabular}

En el análisis de estos datos se evidencia efectivo cambio del grupo experimental contra el grupo control. Es evidente que un $64 \%$ de niños ya no realiza estas acciones en contra de sus compañeros. Sin embargo, un índice de $36 \%$ aún es índice que se debe trabajar con más frecuencia en aula y en casa puesto que los niños ya han grabado cierto tipos de insultos que han aprendido en ambiente de toxicidad. Será más fácil poder llevar esta tarea a cabo si los padres siguen mostrando interés por lograr tener niños felices y saludables.

\section{Tabla 6}

Amenazas a los demás compañeros

\begin{tabular}{ccccc}
\hline & \multicolumn{3}{c}{ GRUPOS POSTEST } \\
\cline { 2 - 5 } Ítem & \multicolumn{2}{l}{$\begin{array}{l}\text { EXPERIMENTAL- } \\
\text { FERRENAFE }\end{array}$} & CONTROL-POMALCA \\
\cline { 2 - 5 } & N $^{\circ}$ & $\mathbf{\%}$ & $\mathbf{N}^{\circ}$ & $\mathbf{\%}$ \\
\hline No & 67 & $74 \%$ & 29 & $32 \%$ \\
Si & 23 & $26 \%$ & 61 & $68 \%$ \\
Total & $\mathbf{9 0}$ & $\mathbf{1 0 0 \%}$ & $\mathbf{9 0}$ & $\mathbf{1 0 0 \%}$ \\
\hline
\end{tabular}

En cuanto a medir los indicadores de agresividad psicológica se obtiene que cuando un niño realiza amenazas no se debe obviar la situación como si fuera normal. Al haberse tratado mediante estratégias claves presentadas en talleres donde el antídoto principal es el amor acomapañado de ensivilidad, amabilidad se logró obtener un indice del $74 \%$ que no presentas este tipo de acciones negativas. Por ello, del $68 \%$ de niños con conductas agresivas con referencia al grupo control se denota disminución del índice a un $26 \%$ en el grupo experimental, observando que es un índice a seguir trabajando. No se debe bajar la guardia en cuanto a temas de conductas agresivas, se debe seguir trabajando en unión los padres con los maestros. Todos somos un equipo.

Gracias a ese estudio se logró el objetivo de determinar la influencia que se obtiene usr la pedagogía de ternura para disminuir las acciones agresivas de los niños de las Instituciones de las que se investigó. Ahora, después de haber obtenido resultados satisfactorios es tarea de las familias de cada niño ser responsables del encubamiento de conductas agresivas hacia sus hijos. Por ello, en la investigacion se citó la participación de los padres, con el fin de trabajar con las familias y ser el apoyo mutuo para el aprendizaje de los niños en base a ternura. Es el hogar donde principalmente se engendran este tipo de acciones porque los padres mayormente al tratar con sus hijos no tienen cuidado con su forma de tratarlos, disciplinarlos, guiarlos y demostrarles siempre el afecto para que tengan un buen desarrollo y crecimiento. 


\section{Discusión}

Este artículo tuvo como objetivo general determinar la influencia del programa pedagogía de la ternura para disminuir la agresividad en niños y niñas. Cruz (2017), invita a la reflexión de la aplicación de la pedagogía de la ternura como herramienta clave para la puesta en práctica en la familia y reforzarlo en el contexto escolar obteniendo convivencias saludables en todos los procesos en las que interactúa el niño. Así, la participación más frecuente de la familia en el quehacer estudiantil de los niños hará niños más capacitados para enfrentar problemas de aprendizaje, de las relaciones sociales y de su yo personal. (p.103). También, los distintos componentes de la pedagogía fomentan el interés por los procesos de aprendizaje y lo encuentran en su maestra el respeto y cariño necesario. En la discusión de los resultados, conforme al entorno agresivo hallado. Se demostró que $57 \%$, 76\% y $66 \%$ de los infantes mostraron comportamientos agresivos físicos, verbales y psicológicos. La aplicación del postest permitió disminuir la agresividad en los niños, a nivel físico entre $23 \%$ a nivel verbal entre $30 \%$ y a nivel psicológico en $26 \%$. Este hallazgo, efectivamente, guarda relación con lo sostenido por Andaur (2017), cuya investigación analizó el claro vínculo y los buenos resultados que traen una enseñanza basada en ternura de parte de los docentes, pues su formación le ha permitido cultivar un fuerte amor por los niños. Si el docente no inspira ni amor ni confianza a los niños. El amor no suele figurar como un componente fundamental de entornos académicos rigurosos. Sin embargo, su importancia resuena tanto en estudiantes como en profesores. Pregúntele a cualquier persona sobre las experiencias de aprendizaje más importantes de su vida y le contarán una historia sobre una relación con un maestro. Contarán los esfuerzos de esa persona para exponerlos a nuevas perspectivas, estimular su reflexión y apoyarlos en los momentos difíciles. Y, si escuchas con atención, escucharás evidencia de la alegría y la satisfacción del maestro en su papel. Estos maestros ocupan su lugar entre los amigos y la familia que dan forma a nuestro sentido de identidad y cómo nos relacionamos con el mundo: las personas que amamos.

Los resultados de la investigación tienen relación a lo mencionado por Bandura (1975), cuya investigación indica que los niños que se encuentran dentro de ambientes tóxicos y agresivos, ellos por imitación tienden a imitar tales conductas y ponerlas en práctica en sus compañeros de clase. Estos efectos dejan en los niños notorias brechas en su memoria y cuyas acciones tienen que ser intervenidas con el fin de frenar estas aconductas negativas. Muchos de nuestros niños y niñas han sido abusados y maltratados, sin saber realmente qué es el amor real ... cómo se ve, cómo suena y cómo actúa, como lo afirma Maya (2015), pues todos nacemos en una familia y allí empezamos a construirnos como personas. No hay niños malos; sólo mal ambiente, mal entrenamiento, mal ejemplo. Seguimos esa filosofía hoy. No es fácil crear ese tipo de ambiente. Los adultos tienden naturalmente a descubrir que los niños son malos. Si llenas esta habitación con mil niños, cualquier adulto no tendría problemas para detectar y describir el comportamiento de los pocos niños que se comportaban mal. Pero se requiere una capacitación extensa para ayudar a los adultos a comprometerse a enfocarse en lo mejor de los niños y describir su buen comportamiento con precisión. Estos resultados muestran que los niños reducen su alto nivel de agresión. Los adultos amorosos te hacen responsable. Si conoces bien a un niño y ves lo mejor en él, se deduce que querrás que esté a la altura de su (mejor yo) haciéndolo responsable. Si vemos que un niño es talentoso en el arte, lo único que puede amar Lo que hacemos es fomentar este talento para que el niño pueda convertirse en el artista que debe ser. Si vemos que los niños pueden comportarse bien algunas veces, debemos ayudarlos a desarrollar esto y aprender a controlarse y comportarse bien la mayor parte del tiempo. Aquí es donde muchos adultos luchan y fracasan en sus relaciones con los niños. ¿Por qué? A menudo, es porque las circunstancias de muchos padres pueden hacer que sea muy difícil responsabilizar a los niños. Se requiere energía, fuerza y un profundo deseo de ayudar a los niños a convertirse en personas que estaban destinadas a ser.

Cuando no responsabiliza a los niños, no los ama en realidad. Por último, la investigación fue realizada como ayuda para todos los niños y niñas que manifestaban acciones negativas en el aula donde gracias a los talleres basados en amor se logró disminuir la agresividad que ellos presentaban teniendo niños más saludables. 


\section{Conclusiones}

Se concluye que el amor es un rasgo de carácter maravilloso para comenzar a aprender si está comenzando el viaje de construir la educación del carácter en el ritmo de su familia. Debido a que los niños no han estado escuchando palabras dulces como un te amo hijo, durante tanto tiempo, pueden comenzar a comprender el sentimiento y las acciones que acompañan a este rasgo de carácter con bastante facilidad después de haber aplicado sesiones y momentos con sus padres llenos de amor. Este conocimiento se puede aplicar a otros rasgos de carácter, como la paciencia o la responsabilidad. Una vez que ven la conexión entre los rasgos y cómo mejoran el bienestary la relación con los demás, hay un efecto dominó y la conciencia de los valores en la vida cotidiana se vuelvemás evidente. Por otro lado, los talleres aplicados en el aula basados en ternura dieron existosos resultados en niños que presentaban conductas agresivas reemplazándolas con acciones positivas con sus demás compañeros generando un ambiente positivo lleno de amory ternura. Además, se evidenció que si los padres se proponen brindarles lo mejor a sus hijos, buscarán la ayuda debida de la mano con los docentes o ayuda profesional que en casos con violencia más extremos requieran de mejor intervención. Las Instituciones educativas en estudio fueron fuente importante para determinar que se necesita urgentemente de más espacios para que los docentes construyan con los niños algo más importante que conocimiento yesel formarynutrir seres humanos llenos de amor.

\section{Referencias}

Andaur, P. (2017). Tipos de conducta agresiva en los niños y niñas de cinco años de la I.E.I. Nacimos para Triunfar, Huancavelica (UniversidadNacional deHuancavelica). http://repositorio.unh.edu.pe/bitstream/handle/UNH/1570/T.A.AQUISEYANA.pdf

Bandura, A. (1975). Aprendizaje Social y Desarrollo de la Personalidad. Editorial Interamericana.

Cavell, T. A.; Mutignani, L. M.; Alfonso, L. \& Marie Smith, A. (2020). Attachment Tendencies, Big 5 Personality Traits, and Self-Efficacy as Predictors of Mentors' Relationships with Aggressive Children. American Journal of Community Psychology, 66(1-2), 130-143. https://www.evidencebasedmentoring.org/how-mentor-traitsaffect-their-relationship-with-aggressive-children/

Cussiánovich, A. (2015). La pedagogía de la ternura. Una lucha por la dignidad y la vida desde la acción educativa. http://rd.udb.edu.sv:8080/jspui/bitstream/11715/885/1/5\%20La\%20Pedagogia.pdf

Cruz, A. (2017). Pedagogía de la ternura en las familias.

Diario Perú21 (2017, 14 de febrero). En el Perú se divorcian 14 mil parejas al año. https://peru21.pe/lima/perudivorcian-14-mil-parejas-ano-63826-noticia/

Ferreyra, L. (2016). Agressividade infantil no cotidiano escolar: recursos e estratégias para o professor da pré-escola. Universidade Estadual Paulista "Júlio de Mesquita Filho". https://repositorio.unesp.br/handle/11449/147129

Juul, J. (2015). Aggression. Why is it necessary for us and our children? Editorial Herder. Alemania.

Maya, A. (2015). Pedagogía de la ternura: Conceptos básicos. Ecoe Ediciones. Costa Rica. https://www.ecoeediciones.com/wpcontent/uploads/2015/08/Pedagog\%C3\%ADa-de-la-ternura-conceptos-b\%C3\%A1sicos.pdf

Ministerio de Educación (2018). Lineamientos para la Gestión de la Convivencia Escolar, la Prevención y la Atención de la Violencia Contra Niñas, Niños y Adolescentes (No. 004-2018-MINEDU). http://repositorio.minedu.gob.pe/bitstream/handle/ MINEDU/6088/Lineamientos para la gestión de la convivencia escolar\%2Cla prevención y la atención de la violencia contra niñas\%2C niñosy adolescentes.pdf

Repo, L. \& Sajaniemi, N. (2015). Prevención del bullying en entornos educativos tempranos: factores pedagógicos y organizativos relacionados con el bullying. Revista europea de investigación sobre educación de la primera infancia. Helsinki.: https://www.redalyc.org/jatsRepo/5216/521654339002/html/index.html

Sánchez, F. (2018). Agresividad infantil y entorno familiar. La albolafia: Revista de Humanidades y Cultura. https://dialnet.unirioja.es/descarga/articulo/6528581.pdf http://repositorio.une.edu.pe/bitstream/handle/UNE/1218/TD CE 1610 S1.pdf

ONU (2017). Informe mundial sobre la violencia contra los niños y niñas. https://violenceagainstchildren.un.org/sites/ violenceagainstchildren.un.org/files/document_files/world_report_on_violence_against_children_sp.pdf

Organización Mundial de la Salud (2019). Prevención de la violencia en la escuela: un manual práctico. Organización Mundial de la Salud. 


\section{Cómo citar este trabajo}

Cajusol Santisteban, C., \& Fernández-Otoya, F. A. (2021). Pedagogía de la ternura para disminuir conductas agresivas en niños. EDUCARE ET COMUNICARE: Revista De investigación De La Facultad De Humanidades, 9(2), 5363. https://doi.org/10.35383/educare.v9i2.583

\section{Financiación}

El presente artículo no cuenta con financiación específica para su desarrollo y/o publicación.

\section{Conflicto de interés}

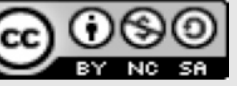

(C) Los autores. Este artículo es publicado por la Revista Educare et Comunicare de la Facultad de Humanidades, Universidad Católica Santo Toribio de Mogrovejo.

Este es un artículo de acceso abierto, distribuido bajo los términos de la Licencia Creative Commons Atribución-NoComercial-CompartirIgual 4.0 Internacional (CC BY- NC-SA 4.0), que permite el uso no comercial, distribución y reproducción en cualquier medio, siempre que la obra original sea debidamente citada. 